NASA Technical Memorandum 102704

\title{
DEVELOPMENT AND TESTING OF METHODOLOGY FOR EVALUATING THE PERFORMANCE OF MULTI-INPUT/ MULTI-OUTPUT DIGITAL CONTROL SYSTEMS
}

Anthony S. Pototzky, Carol D. Wieseman, Sherwood Tiffany Hoadley, and Vivek Mukhopadhyay

\section{August 1990}

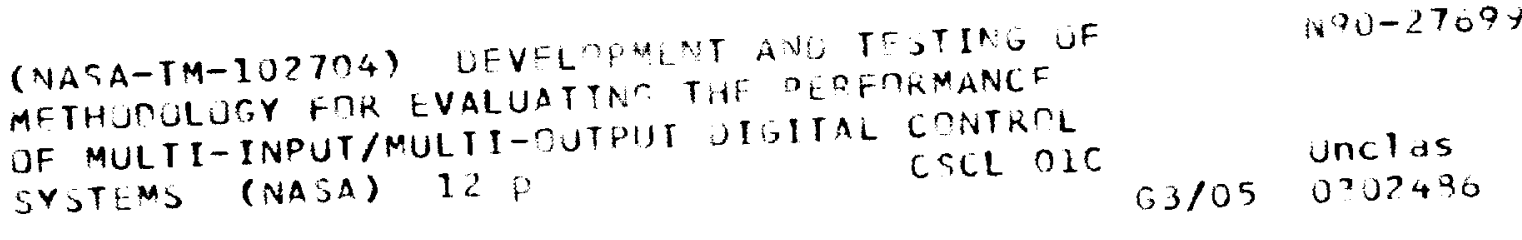

\section{NMSA \\ National Aeronautics and \\ Space Administration}




\title{
DEVELOPMENT AND TESTING OF METHODOLOGY FOR EVALUATING THE PERFORMANCE OF MULTI-INPUT/MULTT-OUTPUT I
}

\author{
Anthony S. Pototzky* \\ Lockheed Engineering and Sciences Company \\ Hampton, Virginia \\ Carol D. Wieseman*, Sherwood Tiffany Hoadley**, and Vivek Mukhopadhyay** \\ National Aeronautics and Space Administration \\ Hampton, Virginia
}

\begin{abstract}
A Controller Performance Evaluation (CPE) methodology for multi-inpul/multi-output digital control systems was developed and tested on an aeroelastic windtunnel model. Modern signal processing methods were used to implement control laws and to acquire time domain data of the whole system (controller and plant) from which appropriate transfer matrices of the control system could be generated. Matrix computational procedures were used to calculate singular values of retumdifference matrices at the plant input and output points to evaluate the performance of the control system. The CPE procedures effectively identified potentially destabilizing controllers, and confirmed the satisfactory performance of stabilizing ones.
\end{abstract}

\section{Nomenclature}

$\begin{array}{ll}\lambda & \text { eigenvalues } \\ \sigma & \text { singular values } \\ \omega & \text { frequency } \\ \operatorname{det}(\cdot) & \text { determinant } \\ \mathbf{G} & \text { plant transfer matrix } \\ \mathbf{H} & \text { controller transfer matrix } \\ \mathbf{I} & \text { identity matrix } \\ \mathbf{N} & \text { number of time segments } \\ \mathbf{n}_{\mathbf{a}} & \text { number of control-law-output actuator commands } \\ \mathbf{n}_{\mathbf{S}} & \text { number of control-law-input sensor } \\ & \text { measurements } \\ \mathbf{S}_{\mathbf{u} \mathbf{X}} & \text { cross-spectrum of excitation to actuator } \\ & \text { commands } \\ \mathbf{S}_{\mathbf{u} \mathbf{u}} & \text { auto-spectra of excitation } \\ \mathbf{S}_{\mathbf{u ~}} & \text { cross-spectrum of excitation to plant responses } \\ \mathbf{X}_{\mathbf{u}} & \text { controller output transfer matrix } \\ \mathbf{Y}_{\mathbf{u}} & \text { plant output transfer matrix }\end{array}$

\section{Subscripts}

$\begin{array}{ll}\mathrm{e} & \text { plant input } \\ \mathrm{u} & \text { command } \\ \mathrm{x} & \text { controller output } \\ \mathrm{y} & \text { plant output }\end{array}$

* Member, AIAA

** Associate Fellow, AIAA

\section{Definitions}

stability -

robustness -

performance - all the poles of the system are on the left-hand side of the complex plane tolerance of system stability to plant uncertainty which can be measured in terms of minimum singular values or gain and phase margins.

performance of the controller is measured in terms of stability and robustness.

\section{Introduction}

Active controls are becoming an increasingly important means to enhance the performance of aircraft. But, because the process of designing multi-input/multioutput (MIMO) digital control laws uses relatively untested theoretical methods, it has become crucial to validate the design methodology through experimentation. For classical single-input/single-output (SISO) control systems, analysis tools such as Nyquist diagrams were developed and used to determine the stability and robustness of the closed-loop system. For MIMO systems, Nyquist techniques are inadequate. Subsequently, analytical methods based on the use of singular values of return-difference matrices at various points in the control loop were developed (references 1 3) to examine the stability and robustness of the control system.

For examining the stability and robustness of MIMO control systems, the plant is excited by some known input. Experimental time-history data are acquired and consist of the excitation and system responses (plant and controller outputs). These time history data are then transformed to frequency domain transfer functions using Fast Fourier Transform (FFT) methods so that transfer matrices and the return-difference matrices can be computed. From these return-difference matrices the singular values are determined to obtain a measure of system stability and robustness. The steps from acquiring the data through interpreting the singular values comprise a methodology referred to as Controller Performance Evaluation (CPE). The methodology is generic in nature and can be used in many types of multi-loop digital 
controller applications including digital flight control systems, digitally controlled spacecraft structures, and actively controlled wind-tunnel models.

In the case of actively controlled wind-tunnel models, flutter testing adds an extra risk because the controller itself can potentially destabilize the model. The on-line near-real time CPE methods were employed to check the stability of the closed-loop system to reduce the risk of damage to the wind-tunnel model and the tunnel.

The present paper describes the implementation of the CPE capability, the structure of the data flow, the signal processing methods used to process the data, and the software developed to generate the transfer functions. In addition, a brief development of the equations used to obtain the open-loop plant, controller transfer matrices, and return-difference matrices are given. Finally, results of applying the CPE methodology to evaluate (in near real-time) MIMO digital flutter suppression systems being tested on the Rockwell Active Flexible Wing (AFW) wind-tunnel model (reference 4) are presented to demonstrate the CPE capability. The AFW wind-tunnel test program is described in references 5 and 6.

\section{Controller Performance Eyaluation}

A simplified block diagram of the basic closed-loop control problem is presented in Figure la. The plant to be controlled is represented mathematically by a frequency domain transfer matrix, G, with outputs y and inputs $e$. The controller is represented mathematically with a transfer matrix, $H$, with inputs $y$ and outputs $x$. External excitation $u$ is used to excite the system in a specified fashion. This excitation is used to derive transfer functions between outputs and inputs in either open- or closed-loop systems. The open-loop system is one in which the control law outputs (commands required for controlling plant response) are not fed back into the system; i.e., the switch depicted in figure $1 b$ is open.

Controller performance evaluation is a two-mode, four-step process. The two modes are open- and closedloop, and each mode can be broken into two steps. The process is outlined conceptually for the flutter suppression system application as follows:

\section{Open-1000}

Step 1: Verify the controller, $\mathbf{H}$, by comparing with the designed control law transfer matrix.

Step 2: Predict closed-loop performance based on the open-loop performance to determine whether the control law will stabilize or destabilize the system when the loop is closed.

\section{Closed-loop}

Step 1: Determine the stability margins of the closed-loop system during the closed-loop testing by evaluating the singular values of return-difference matrices, $(\mathbf{I}+\mathbf{G H})$ and (I+HG).

Step 2: Determine open-loop plant stability during the closed-loop testing to determine the open-loop flutter boundary.

\section{CPE Computations}

The CPE computations involve generating frequency domain transfer functions of plant outputs, $y$, due to an excitation, $u$, and control law commands, $x$, due to the same excitation. Fast Fourier Transform (FFT) techniques are used to calculate these functions. The controller, $\mathbf{H}$, and the return-difference matrices and their singular values are then calculated using matrix operations. The computations are described in the following paragraphs. Figure 2 is a flowchart which outlines the CPE procedures.

\section{Transfer Functions}

The method used to compute transfer functions is described in reference 7 . The method was extended in the present study to include additional data-windowing capabilities and overlap averaging. Windowing capabilities include ramp-in/ramp-out, Hanning, cosine taper, and cosine bell. The overlap-averaging capability allows long time histories to be partitioned into shorter time spans, taking advantage of long periods of time history data to average out noise. A zero-fill capability is available to zero fill time history data to an exact increment of a power of two needed for FFT computations. The overlap-averaging capability with zero-fill provided optimum use of the time history data which were obtained.

The controller-output transfer matrix, $x_{u}$, is the matrix of ratios of the cross-spectra of the controller outputs, $x$, due to the excitations, $u$, to the auto-spectra of the excitations, $u$; all spectra are obtained from the FFT's of the time histories. Each element of the transfer matrix is given by:

$$
\left(\mathbf{x}_{\mathbf{u}}(\omega)\right)_{\mathbf{J}}=\frac{\sum_{\mathbf{m}=1}^{N}\left(s_{\mathbf{u}_{i} \mathbf{x}_{j}}(\omega)\right)_{m}}{\sum_{m=1}^{N}\left(s_{u_{\mid} u_{j}}(\omega)\right)_{m}}
$$


where $N$ is the number of time history segments. Similarly, each element of the plant-output transfer matrix, $Y_{u}$, is given by equation (2).

$$
\left(Y_{u}(\omega)\right)_{\mathbb{J j}}=\frac{\sum_{m=1}^{N}\left(s_{u_{i} y_{j}}(\omega)\right)_{m}}{\sum_{m=1}^{N}\left(s_{u_{j} u_{j}}(\omega)\right)_{m}} .
$$

The two matrices, $X_{u}$ and $Y_{u}$, are the basis of all the subsequent $\mathrm{CPE}$ computations.

\section{CPE Procedures}

Qpen-Loop Case. Both transfer matrices, $\mathbf{x}_{u}$ and $Y_{u}$, are obtained from a control system in which the loop is open at the controller output. Referring to figure $1 b$, it can be observed that the elements of the plant transfer matrix, $\mathbf{G}$, (dimensioned $\mathrm{n}_{\mathbf{S}}$ by $\mathrm{n}_{\mathbf{a}}$ ) are defined by equation (2), because, in this case,

$$
\mathbf{G}(\omega)=\mathbf{Y}_{\mathbf{u}}(\omega)
$$

The elements of the open-loop controller-plant transfer matrix, HG, (dimensioned $n_{a}$ by $n_{a}$ ) are defined by equation (1), since

$$
\text { HG }(\omega)=\mathbf{X}_{\mathbf{u}}(\omega) \text {. }
$$

A solution for the controller transfer matrix, $H$, (dimensioned $n_{\mathbf{a}}$ by $n_{S}$ ) can be obtained from equations (3) and (4),

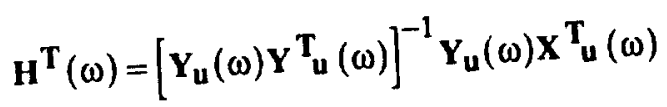

If $n_{a}$ is greater than $n_{s}$, then this is actually a least square solution. For the special case of $n_{a}$ equal to $n_{s}, Y_{u}$ is a square matrix and a simplified and more computationally efficient form of equation (5) shown in equation (6) can be used.

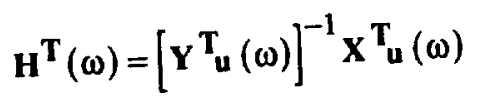

To perform the first step of the open-loop CPE, the resulting controller transfer matrix, $\mathbf{H}$, computed with either equation (5) or (6) is compared with the designed control law transfer matrix to verify the implementation of the controller. Specifically, the transfer functions are compared for each output/input pair.

To perform the second step in the open-loop CPE (predicting closed-loop performance based on the openloop performance to decide whether the control law will stabilize or destabilize the system when the loop is closed), it is convenient with a MIMO system to evaluate stability and robustness by examining the minimum singular values of the return-difference matrices at the plant input

$$
\sigma_{\min }(\mathbf{I}+\mathbf{H G})
$$

and the plant output

$$
\sigma_{\min }(\mathbf{I}+\mathbf{G H})
$$

System instabilities occur at frequencies when the minimum singular value becomes zero. Therefore the proximity to zero indicates where the system is prone to go unstable and provides a quantitative measure of robustness. Reference 3 contains a derivation which relates guaranteed gain and phase margins to minimum singular values. This relationship is shown in Figure 3 of the present paper, which is a reproduction of figure 2 from reference 3 , and will be referred to later when discussing results.

The matrix product $\mathbf{H G}$ is obtained from equation (4). The matrix product $\mathbf{G H}$ can be obtained from equations (3) and (5) or (6) since

$$
\mathbf{G H}(\omega)=\mathbf{G}(\omega) \mathbf{H}(\omega)
$$

The maximum singular values of the return-difference matrices at the plant input,

$$
\sigma_{\max }(\mathbf{I}+\mathbf{H G})
$$

and the plant output

$$
\sigma_{\max }(\mathbf{I}+\mathbf{G H})
$$

were also determined. The ratio of the maximum to minimum singular values of a return-difference matrix is the condition number. If a minimum singular value approaches zero (has low stability margins), the size of the condition number, especially when it is much larger than one, becomes an important indicator of the uncertainty in the measure of system stability; ie. large condition numbers indicate that the predicted stability margin is very uncertain.

Singular values of the return-difference matrices give a conservative measure of the system stability margins. The determinant, det (I+HG), and the magnitude of the minimum eigenvalue, $\left|\lambda_{\min }(\mathbf{I}+\mathbf{H G})\right|$, of the returndifference matrices are less conservative measures of stability. The minimum eigenvalue at the plant input and the plant output are identical. Likewise, the determinants are also identical. In general, the propertics of the magnitude of the minimum eigenvalues are similar to the properties of the minimum singular values; both are measures of how close the returndifference matrices are to a singularity. The locus of the determinant of the return-difference matrix as a function of frequency has properties similar to those of a Nyquist diagram for SISO control systems. If the open-loop 
system is stable, an encirclement of the critical point (the origin) for $\operatorname{det}(\mathbf{I}+\mathbf{H G}(\omega))$ indicates that the controller is destabilizing. Furthermore, the proximity of the determinant locus to the critical point is a direct indication of how near the control system is to an instability.

Closed-Loop Case. The objective of step 1 is to obtain transfer matrices HG and GH so that singularvalue, eigenvalue and determinant calculations can be performed on the return-difference matrices. The difference between closed-loop and open-loop computations is that the transfer matrices, $X_{u}$ and $Y_{u}$, are obtained from the closed-loop system. During closed-loop testing, the plant transfer matrix is determined from equation (8)

$$
\mathbf{G}^{\mathbf{T}}(\omega)=\left[\mathbf{I}-\mathbf{X}_{\mathbf{u}}^{\mathbf{T}}(\omega)\right]^{-1} \mathbf{Y}_{\mathbf{u}}^{\mathbf{T}}(\omega)
$$

and the open-loop controller-plant transfer matrix is determined from equation (9)

$$
[\mathbf{H G}]^{\mathbf{T}}(\omega)=\left[\mathbf{I}-\mathbf{x}_{\mathbf{u}}^{\mathbf{T}}(\omega)\right]^{-1} \mathbf{x}_{\mathbf{u}}^{\mathbf{T}}(\omega)
$$

Noting that the quantity

$$
\left[\mathbf{I}-\mathbf{X}_{\mathbf{u}}^{\mathbf{T}}(\omega)\right]^{-1}
$$

is in both equations, transfer matrices $\mathbf{G}$ and $\mathbf{H G}$ are obtained simultaneously using matrix partitioning. The controller transfer matrix, $\mathbf{H}$ is calculated using either equation (5) or equation (6), as in the open-loop case.

The singular-value plots and eigenvalue plots are interpreted the same way for closed-loop testing as they were for open-loop testing. However, care must be taken in interpreting the determinant plots. If the open-loop plant is unstable with one right-hand pole (for positive frequencies), then the plot will show no net encirclement about the origin for a stable closed-loop system. As with the open-loop case, the proximity of the determinant locus to the critical point and the proximity of the minimum singular values to zero are used as measures of closed-loop stability.

To obtain results for step 2 of the closed-loop mode, the inverted maximum singular value of the plant transfer matrix, $\mathbf{G}$, as given by,

$$
\frac{1}{\sigma_{\max }(\mathbf{G}(\omega))}
$$

was an excellent indicator of poles in the proximity of the imaginary axis. The frequency of instability was determined by where the inverse maximum singular value approaches zero. Tracing values of closest approach was a useful way of determining the open-loop plant flutter boundary with respect to some changing test condition, such as dynamic pressure.

\section{Summary of Elutter-Suppression Testing}

During flutter suppression testing, the control systems were operated in both open-loop and closed-loop modes. For the purpose of maintaining both model and tunnel safety, each candidate control law was initially tested open loop to insure that the control law itself would not destabilize the wind-tunnel model during closed-loop tests. The feedback was digitally switched open at the control law output point and the response data was collected at the control law input and the control law output locations. The stored data are the excitation, $u$, the control law output, $x$, and the control law input, $y$, depicted in figure 1. The appropriate transfer functions were generated from these responses and then the CPE capability was exercised to predict the stability of the closed-loop system (figure 2). If the control law was predicted to be stable, the switch was closed and the closed-loop flutter suppression (FS) testing for that candidate control law commenced. During the closed-loop portion of the test, the excitations were inserted and response data was taken at the same test conditions as the initial open-loop tests. At each test point, stability margins and open-loop plant stability were determined before proceeding to the next test point.

Figure 4 shows the plant and FS control laws. For this model, separate FS control laws were required for controlling symmetric and antisymmetric flutter modes simultaneously. The CPE excitation was input to the control surfaces either symmetrically or antisymmetrically depending on which control law was being evaluated. The responses, $y$, were summed or differenced before saving.

\section{Description of the CPE Imolementation}

\section{Hardware}

The digital controller not only provided basic control and flutter suppression of the model, but was the source for the excitations needed for CPE. The excitation was generated digitally and added to the control law actuator commands. The digital excitation, actuator commands, and sensor measurements used by the control law were stored and then transferred to a SUN 3/160 computer with a SKY Warrior II array processor board (capable of up to 8K FFT computations) where the FFT computations were performed. The matrix computations to obtain the singular values of return-difference matrices were also performed on the SUN 3/160.

\section{Software}

All the digital controller software was written in the high level $C$ programming language, except for the 
commands required to perform the actual calculations on the array processor. Operation code command blocks were generated for these. The FFT's of the time histories of the excitations and the responses, and the transfer functions, were computed in a Fortran-77 program, optimized to take advantage of the vector-processing capabilities on the array processor. The detailed CPE analysis capability was implemented using MATLAB software operations (reference 8).

\section{Results and Discussion}

Both SISO and MIMO flutter suppression control laws were designed for the AFW wind-tunnel model. During the wind-tunnel test the FS control laws were successfully tested and their performance evaluated using the CPE capability presented in this paper.

The data for performing CPE was obtained by exciting, one at a time, all pairs of control surfaces used by the control law. The surfaces were excited symmetrically or antisymmetrically (corresponding to either the symmetric or the antisymmetric FS control laws) with a logarithmic sine sweep. In most cases the sweep duration was 150 seconds over a frequency range from 4 to $35 \mathrm{~Hz}$. The excitation, the plant responses as measured by various accelerometers, and the controller outputs, corresponding to actuator commands, were saved. While these stability computations were being performed for the symmetric controller, the antisymmetric excitations were performed and the transfer matrices generated. Final results and plots were available approximately 3 minutes after the last sweep was performed.

The first five seconds of a sample excitation, resulting actuator commands and accelerometer responses are shown in figure 5 . The quantization levels of the digital controller can be noted by the step-like behavior of the time histories for the actuator commands. A typical transfer function at one test condition is shown in figure 6.

\section{SISO Control Law}

Typical CPE results for a symmetric SISO control law (control law 2 of reference 6 ) obtained during the closed-loop wind-tunnel tests are shown in figures 7 and 8. Because, for SISO control laws, eigenvalue and singular value plots are identical, only one plot is shown. Also, for SISO control laws, the determinant plot is identical to the Nyquist plot. The determinant plot in figure 7 shows no encirclement about the origin (the critical point) at a dynamic pressure of $200 \mathrm{psf}$ where the open-loop plant is known to be stable. Figure 8 shows the CPE results of a closed-loop system where the plant is unstable. Since the minimum singular values are greater than zero and there is no net encirclement of the critical point, these plots indicate that the controller is stabilizing the plant. Using the minimum singular value from figure 7 , guaranteed stability margins can be obtained from the universal gain and phase diagram of figure 3. Since the $\sigma_{\min }=0.37$, the gain margin for zero phase margins are approximately $-2.7 \mathrm{db}$ and $+4.0 \mathrm{db}$ and for a 20 degree phase margin, the gain margins are $-0.7 \mathrm{db}$ and $+2.05 \mathrm{db}$.

\section{Mimo Control Law}

Results for a MIMO FS control law design (control law 1 of reference 6) are presented next. Since the plots of the maximum and minimum singular values of the return-difference matrices at the plant input and output locations along with the eigenvalue plot for the MIMO system are not identical as in the SISO control system, all three plots are shown in figure 9. In the upper right plot of figure 9 , the ratio of the maximum to the minimum singular value (ie. condition number) is large in the vicinity of $10 \mathrm{~Hz}$. As discussed previously large condition numbers indicate uncertainty in the computation of the minimum singular values. The determinant plot (lower right) shows an encirclement of the origin where the open-loop plant is known to be stable, thus indicating that the MIMO control law would be destabilizing. Both the minimum singular value and eigenvalue plots show noticeably low stability margins near $20 \mathrm{~Hz}$. Upon further investigation, one of the elements of this destabilizing controller transfer matrix was examined and is shown in figure 10 . The figure shows a peak magnitude close to $20 \mathrm{~Hz}$.

\section{Elutter Prediction}

A plot of the inverse maximum singular values of the plant transfer matrix computed from closed-loop tests for a dynamic pressure of $200 \mathrm{psf}$ is shown in figure 11. The frequencies at which the two local minima (approximately 8.5 and $11 \mathrm{~Hz}$ ) of inverse maximum singular value curve occur are indicated by the arrows. These frequencies correspond to the frequencies of the modes which coalesce to create flutter. These frequencies were determined for many dynamic pressures and figure 12 shows a plot of the two frequencies as a function of dynamic pressure. The dynamic pressure at which the two curves appear to coalesce indicates a potential point for open-loop flutter.

\section{Conclusions}

A Controller Performance Evaluation (CPE) methodology was developed to evaluate the performance of multivariable, digital control systems. The method was used and subsequently validated during the windtunnel testing of an aeroclastic model equipped with a digital flutter suppression controller. Through the CPE effort a wide range of sophisticated real-time analysis tools were developed. These tools proved extremely useful and worked very well during wind-tunnel testing. Moreover, results from open-loop CPE were the sole criteria for beginning closed-loop testing. In this way, CPE identified potentially destabilizing controllers before 
actually closing the loop on the control system, thereby avoiding catastrophic damage to the wind-tunnel model or the tunnel. Open-loop plant transfer functions derived from CPE computations were used to redesign and improve control laws. CPE results also proved useful in determining open-loop plant stability during closed-loop test conditions.

\section{Acknowledgements}

The authors wish to thank William M. Adams, Jr. for his continued insistence on the need for an on-line real-time controller performance evaluation capability and to acknowledge his original outline for the CPE procedures. We would also like to thank Boyd Perry III for his coordination and guidance in carrying through the CPE effort. We would also like to acknowledge Matt Fox who did much of the MATLAB coding of the CPE procedure.
6. Perry III, B.; Mukhopadhyay, V.; Tiffany Hoadley, S.; Cole, S.R.; Buttrill, C.S. and Houck, J.A.: Digital-Flutter-Suppression-System Investigations for the Active Flexible Wing Wind-Tunnel Model. NASA TM-102618, AIAA Paper 90-1074, Presented at the 31th AIAA Structures, Structural Dynamics and Materials Conference in Long Beach, California, April 1990.

7. Adams, Jr., William M.; Tiffany, Sherwood H.; and Bardusch, Richard E.: Active Suppression of an "Apparent Shock Induced Instability", presented at the AIAA 28th Structures, Structural Dynamics, and Materials Conference, AIAA Paper 87-0881CP, April 6-8, 1987, Monterey, California.

8. PRO-MATLAB User's Guide, The MathWorks Inc.; 21 Eliot Street; South Natick, MA 01760.

\section{References}

1. Mukhopadhyay, V. and Newsom, J.R.: A Multiloop System Stability Margin Study Using Matrix Singular Values. Journal of Guidance, Control, and Dynamics, vol. 7 no. 5, Sept.October 1984, pp. 582-587.

2. Mukhopadhyay, V. and Newsom, J. R.: Application of Matrix Singular Value Properties for Evaluating Gain and Phase Margins of Multiloop Systems. AIAA Guidance, Control, and Dynamics Conference, AIAA Paper 82-1574, August 9-11, 1982, San Diego, California.

3. Mukhopadhyay, V.; Pototzky, A. S. and Fox, Matthew: A Scheme for Theoretical and Experimental Evaluation of Multivariable System Stability Robustness. Paper presented at the 1990 American Control Conference, May 23-25, 1990, San Dicgo, California.

4. Miller, G.D.: Active Flexible Wing (AFW) Technology. AFWAL TR-87-3096, Feb. 1988.

5. Noll, T.; et al.: Aeroservoelastic Wind Tunnel Investigations Using Active Flexible Wing Model - Status and Recent Accomplishments. NASA TM-101570, AIAA Paper 89-1168, Presented at the 30th AIAA Structures, Structural Dynamics and Materials Conference in Mobile, Alabama,
April 1989.

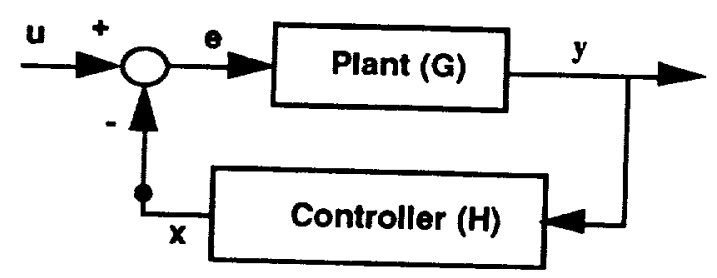

a) Basic closed-loop control problem.

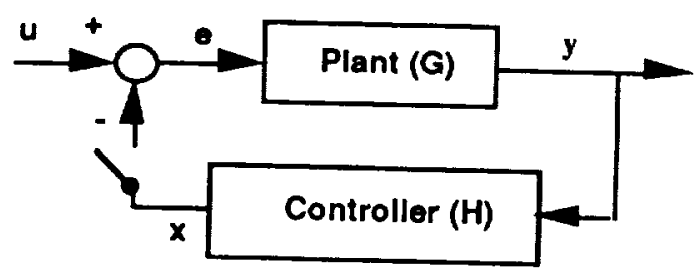

b) Open-loop control problem.

Figure 1. Controller-Plant diagrams depicting the control problem. 


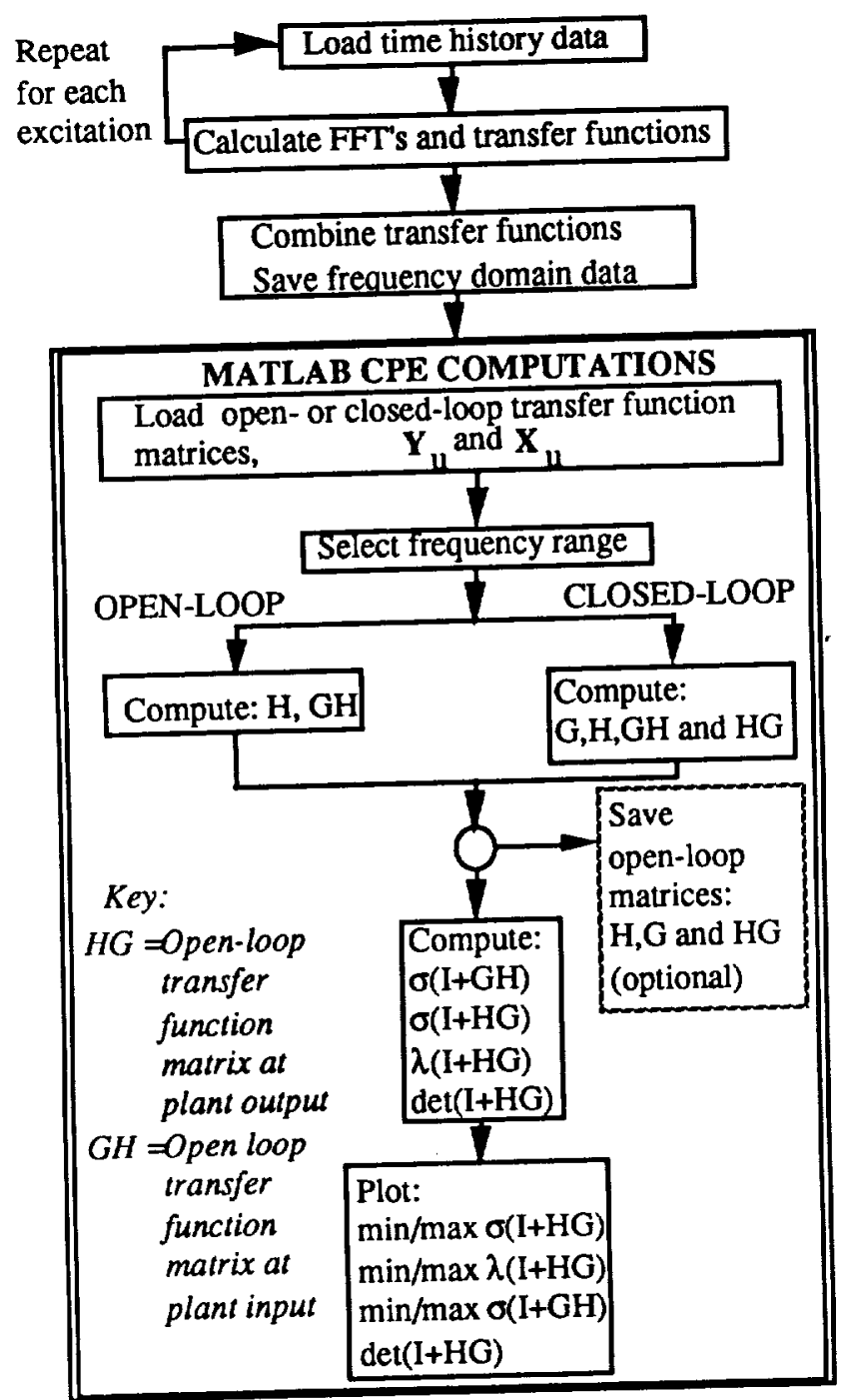

Figure 2. Flowchart of CPE procedures.

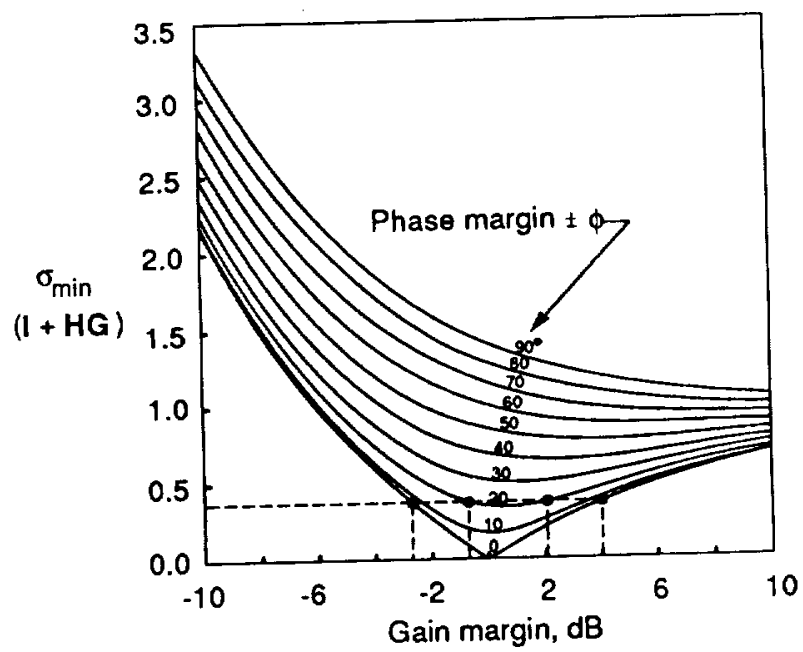

Figure 3. Universal gain and phase margin diagram.

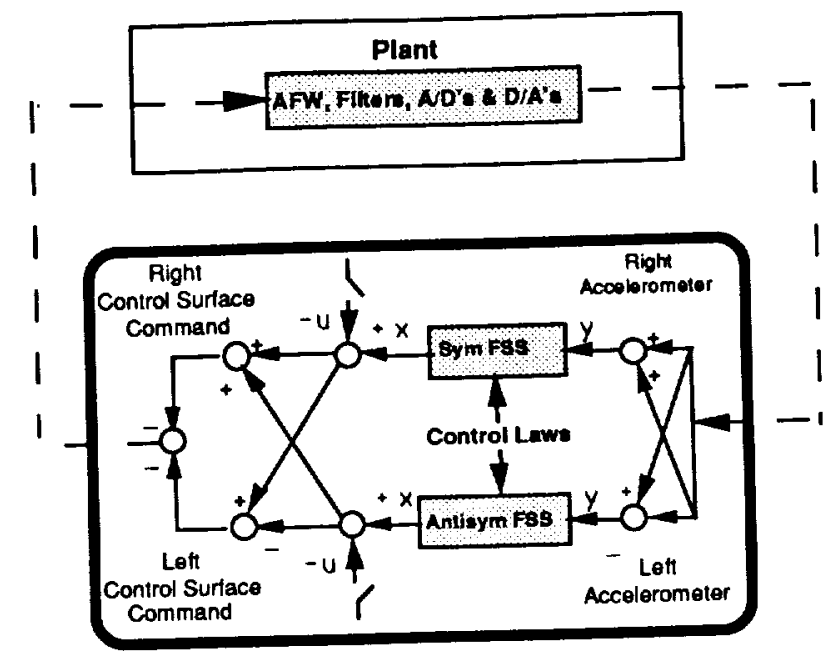

Figure 4. Flutter control law implementation.

a)

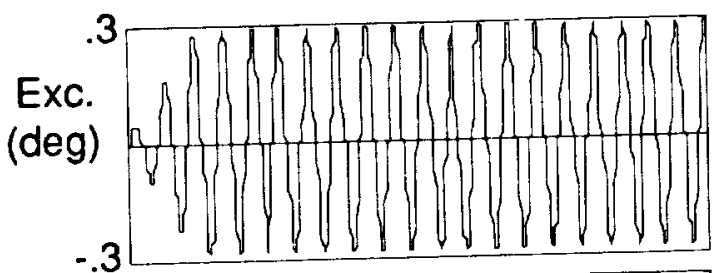

b)

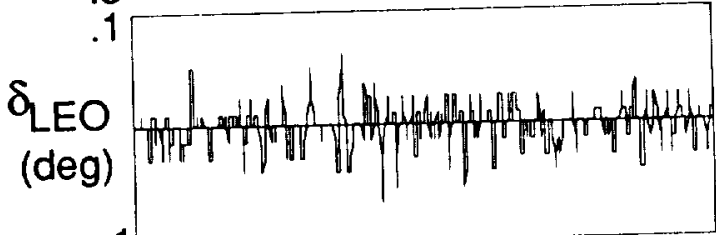

c)

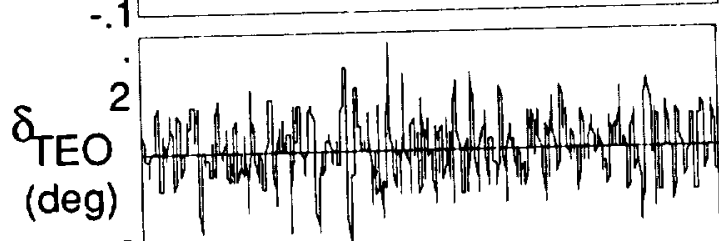

d)

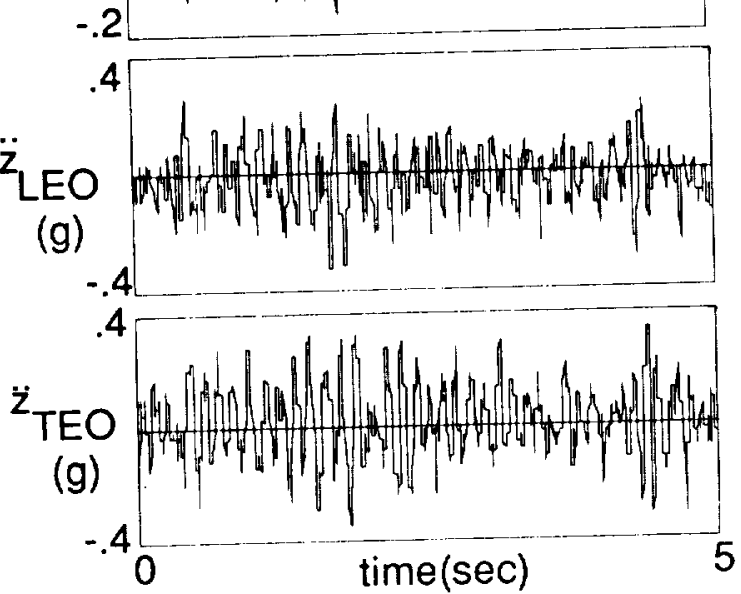

Figure 5. Time histories of sample excitation, actuator commands and accelerometer responses. 


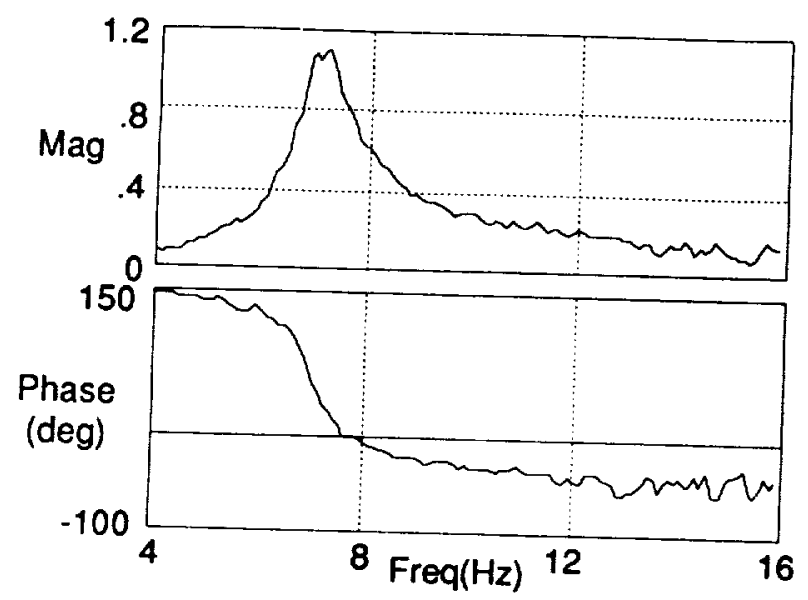

Figure 6. Typical open-loop plant output transfer function.

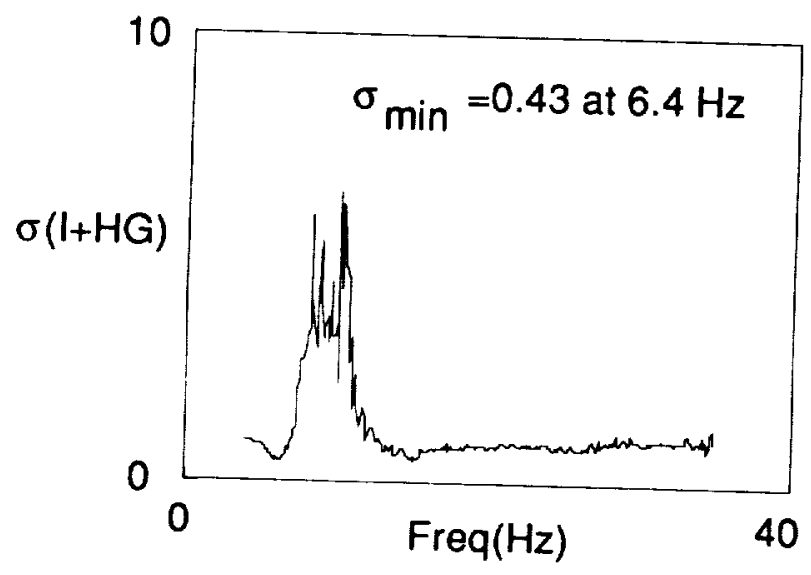

a) Singular value plot.

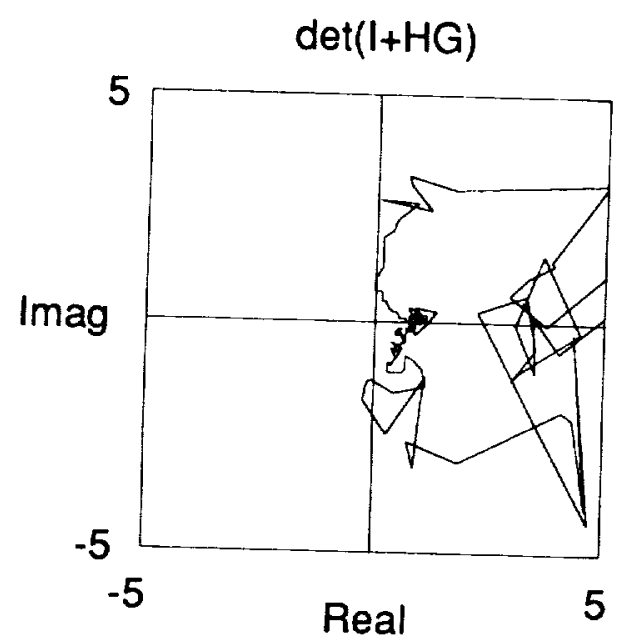

b) Determinant plot.

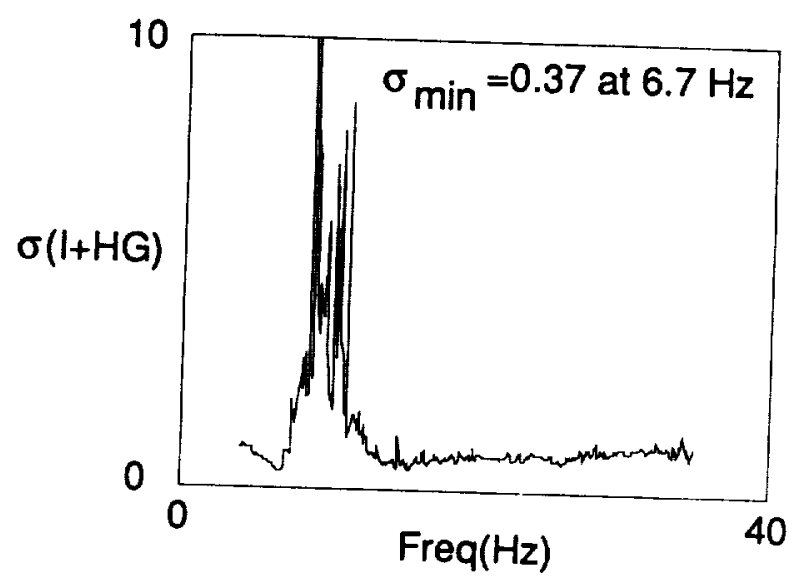

a) Singular value plot.

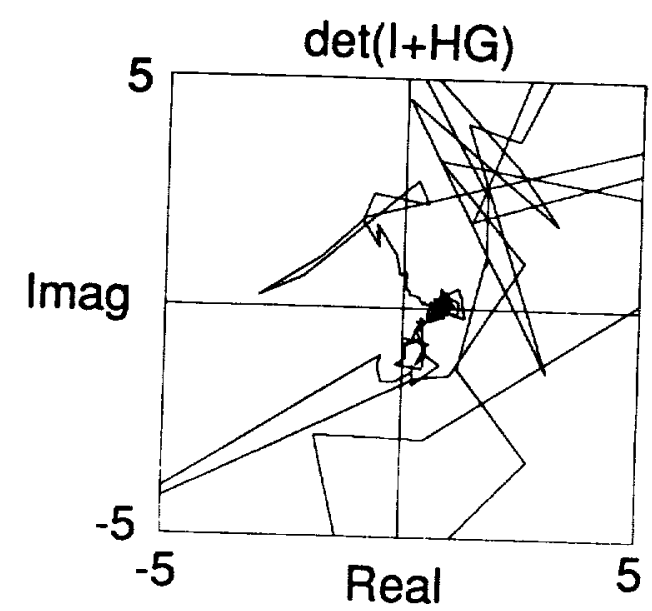

b) Determinant plot.

Figure 8. Closed-loop CPE results for a symmetric SISO control law (open-loop plant is unstable), $M=0.44, q=250$ psf.

Figure 7. Closed-loop CPE results for a symmetric SISO control law (open-loop plant is stable), $M=0.39, q=200$ psf. 

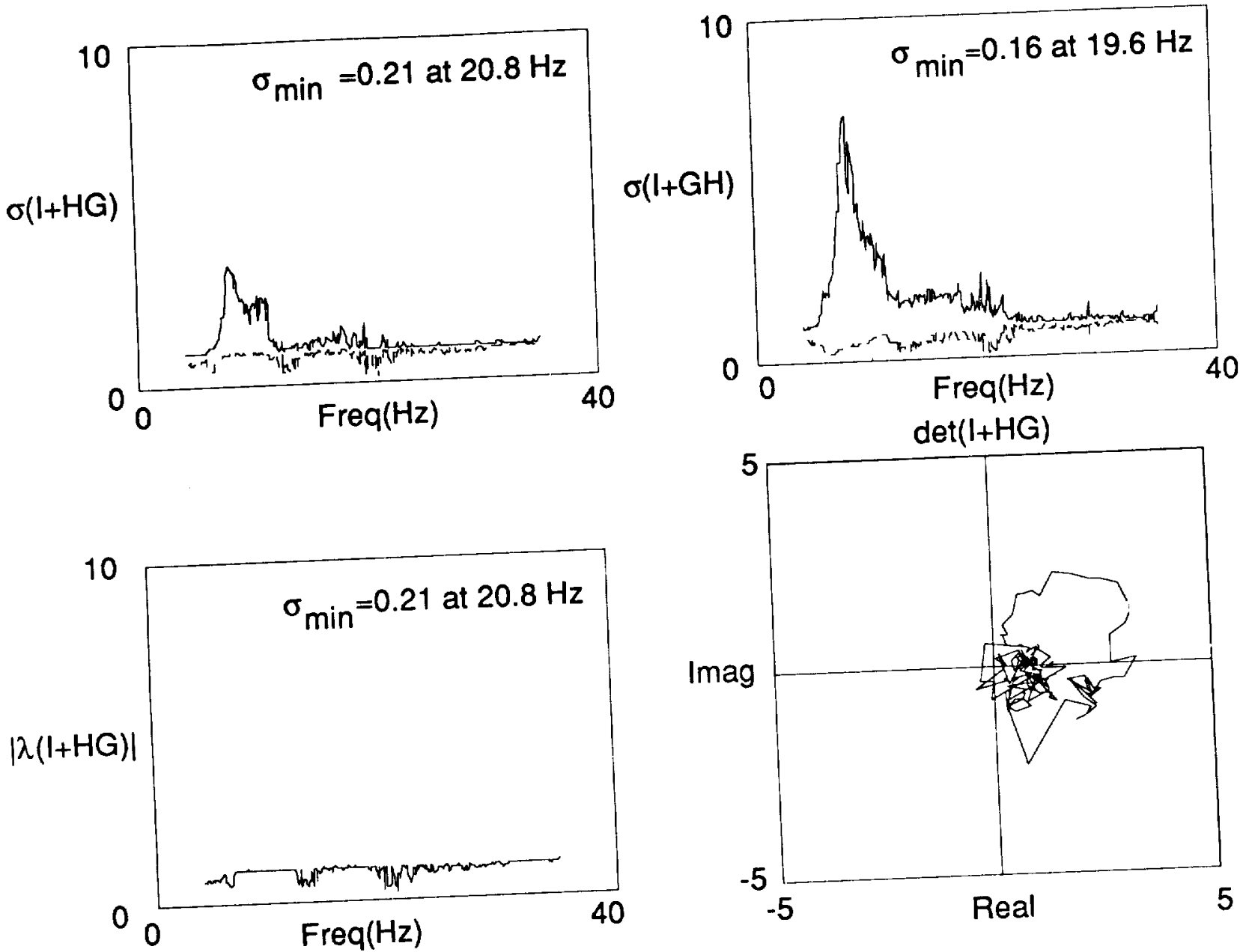

Figure 9. Open-loop CPE results for a symmetric MIMO control law (open-loop plant is stable), $\mathrm{M}=0.36$, $\mathrm{q}=175 \mathrm{psf}$.

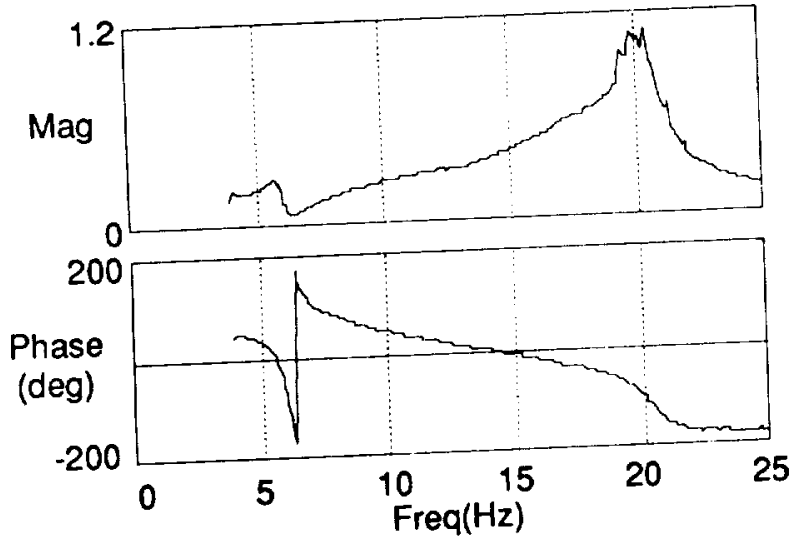

Figure 10. One element of destabilizing controller transfer matrix, $\delta_{\text {TEO }} / \ddot{z}_{\text {TEO }}$.

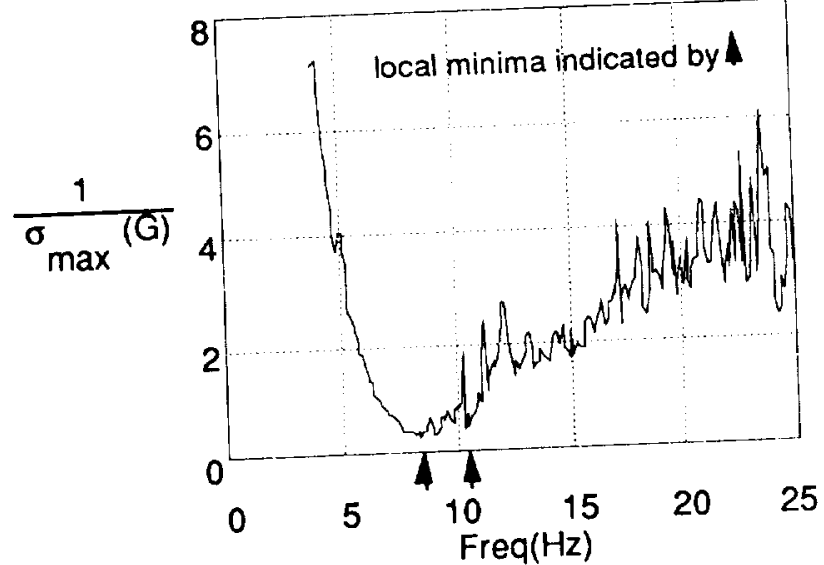

Figure 11. Plot of inverse maximum singular values of the symmetric plant transfer matrix, $q=200$ psf. 


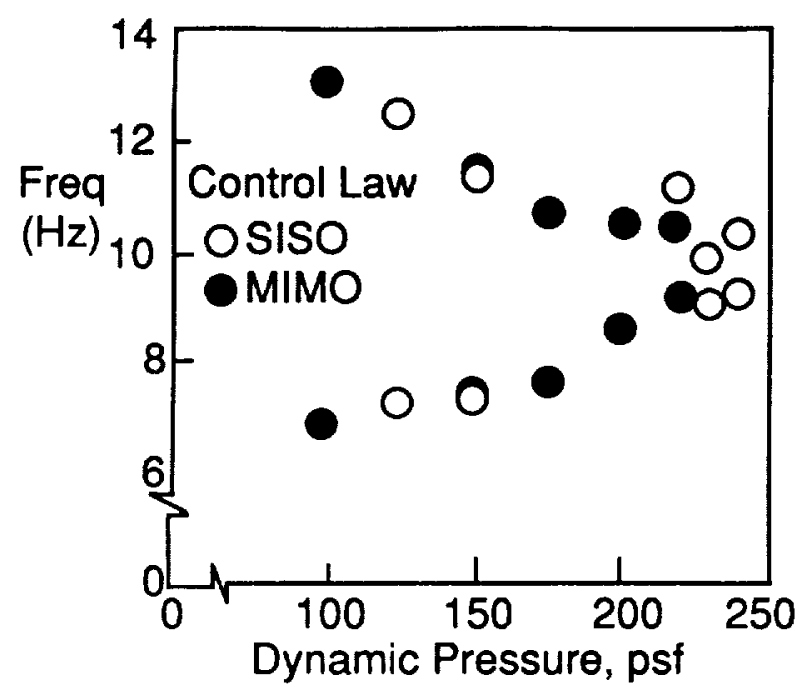

Figure 12. Frequencies of modes contributing to symmetric flutter determined from inverse of maximum singular values. 


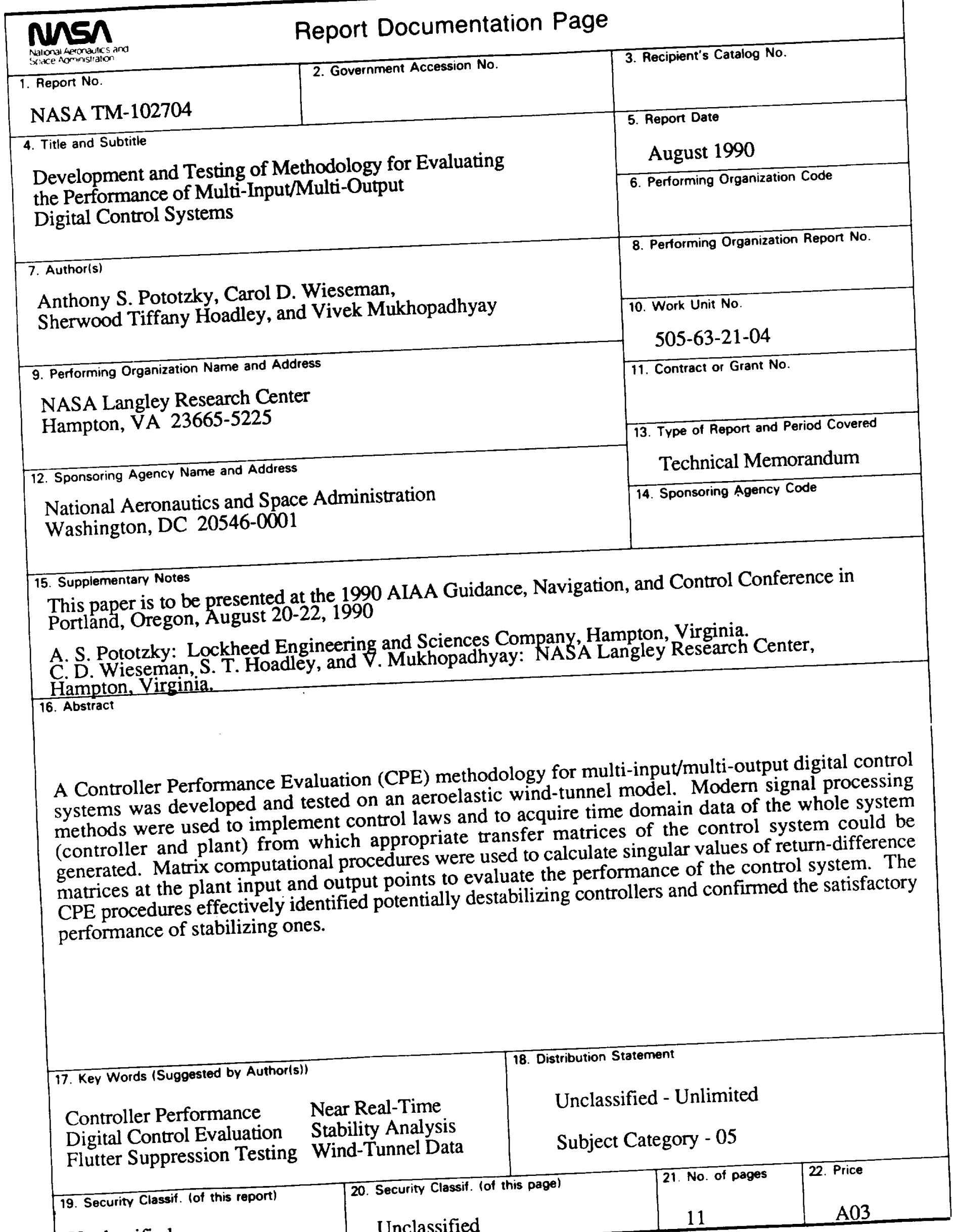

Unclassified

Unclassified 
